\title{
Randomized trial of inhaled fluticasone propionate in chronic stable pulmonary sarcoidosis: a pilot study
}

\author{
R.M. du Bois*, P.M. Greenhalgh ${ }^{+}$, A.M. Southcott*, N.Mcl. Johnson ${ }^{+}$, T.A.J. Harris ${ }^{\#}$
}

\begin{abstract}
Randomized trial of inhaled fluticasone propionate in chronic stable pulmonary sarcoidosis: a pilot study. R.M. du Bois, P.M. Greenhalgh, A.M. Southcott, N.Mcl. Johnson, T.A.J. Harris. (C) ERS Journals Ltd 1999.

ABSTRACT: Pulmonary sarcoidosis is a disease in which the pathological processes are distributed along lymphatic pathways, particularly those around the bronchovascular bundles. Delivery of disease-modulating drugs by the inhaled route is therefore an attractive option. The aim of this study was to determine the efficacy of inhaled fluticasone propionate $2 \mathrm{mg} \cdot \mathrm{day}^{-1}$ in adults with stable pulmonary sarcoidosis.

Forty-four adult patients ( 22 from each centre) were enrolled from outpatient clinics in two London teaching hospitals in a two centre, double-blind, randomized, placebo-controlled trial.

Primary end points were home recordings of peak expiratory flow rate (PEFR), forced expiratory volume in one second (FEV1), and forced vital capacity (FVC). Secondary end points were symptom scores, use of rescue bronchodilator medication, and clinic values for PEFR, FEV1, FVC, forced mid-expiratory flow (FEF25-75\%), diffusion capacity of the lung for carbon monoxide $(D L, C O)$, and total lung capacity (TLC).

Symptom scores of cough, breathlessness and wheeze were lower in the active treatment group, but this did not reach statistical significance, and a general health perception assessment (Short Form (SF)-36) showed a difference between active and placebo treatment. No significant differences were found between the two groups in any physiological outcome measure. No new adverse reactions were detected.

The results of this pilot study do not show an objective benefit of inhaled fluticasone propionate in pulmonary sarcoidosis where the disease is stable and is controlled without the use of inhaled corticosteroids.

Eur Respir J 1999; 13: 1345-1350.
\end{abstract}

\begin{abstract}
*Respiratory Directorate, Royal Brompton Hospital, London, UK. ' Dept of Thoracic Medicine, Whittington Hospital, London, UK. "Glaxo Group Research, Uxbridge, Middlesex, UK.
\end{abstract}

Correspondence: R.M. du Bois

Royal Brompton Hospital

Sydney Street

London SW3 6NP

UK

Fax: 441713518336

Keywords: Fluticasone dipropionate inhaled corticosteroids

sarcoidosis

Received: August 131997

Accepted after revision January 301999
Sarcoidosis is a chronic, multisystem, inflammatory disorder of unknown aetiology characterized by the presence of noncaseating granulomata, in the lungs and other organs [1]. There is some evidence that this intense CD4+ T-cell immune response is driven by a persistent antigen, perhaps a mycobacterium [2]. The natural history of sarcoidosis is variable but is progressive in $\sim 25 \%$ of patients. Systemic steroids are widely used, and appear to be effective, in the treatment of radiographically determined stage II and III pulmonary sarcoidosis, and in stage I patients with deteriorating lung function [3-9], but the adverse risk:benefit ratio of prolonged systemic steroid therapy has prompted a search for alternative treatments.

Uncontrolled clinical trials have suggested that inhaled steroids may favourably influence the course of acute pulmonary sarcoidosis in selected patients [10-12], an impression which has been confirmed in placebo-controlled pilot studies $[3,13]$. A double-blind randomized controlled trial of high-dose inhaled budesonide in newlydiagnosed pulmonary sarcoidosis (within six months of diagnosis), in which 47 patients were randomized, showed significant improvements in symptom scores and inspiratory vital capacity, but not in serum angiotensin converting enzyme (ACE) levels, transfer factor of the lung for carbon monoxide $(T \mathrm{~L}, \mathrm{CO})$ or chest radiograph appearance, after six months of treatment [14]. A second study shows no difference between budesonide-treated and placebo in a number of outcome measures [15].

No previous randomized trial has looked specifically at inhaled steroids in chronic persistent pulmonary sarcoidosis, and the results of a large open trial in chronic sarcoidosis in India were disappointing [16]. A recent review concluded that "it appears to be especially difficult to find criteria for the selection of patients who might respond to this form of therapy" [17].

Fluticasone propionate (FP) is a relatively new topically active synthetic steroid which is very poorly absorbed from the gastrointestinal tract and which undergoes first pass metabolism [18]. Several large randomized trials have shown that FP is effective and safe in seasonal and perennial rhinitis [19], and in both mild [20], moderate and severe asthma $[21,22]$. FP has twice the topical antiinflammatory potency of budesonide (BUD) and beclomethasone dipropionate (BDP), with a lower potential for adrenal suppression at clinically equivalent doses [23]. In vitro studies have demonstrated that FP powerfully inhibits neutrophil function and reduces the chemotactic effect of lung secretions [24], suggesting that it may have 
a role in the prevention of immune-mediated tissue damage in pulmonary sarcoidosis.

The present study was designed to test the hypothesis that high-dose inhaled FP improves (or delays the progression of) chronic pulmonary sarcoidosis as measured by symptoms (breathlessness, wheeze, cough, and sputum), ability to perform daily activities, and objective measures of lung function both at home and in the laboratory. It was of particular interest to assess the effect of FP on airflow obstruction in chronic persistent sarcoidosis. The study was approved separately by the local ethical committees of the participating hospitals; all patients gave written consent before being randomized.

\section{Patients and methods}

The study was a two centre, randomized, placebo-controlled trial. Inclusion criteria were: 1) outpatients of either sex aged 18-65 yrs; 2) persistent pulmonary sarcoidosis diagnosed at least 1 yr previously, on the basis of clinical evaluation and chest radiography showing typical features of the condition and confirmed by bronchial or transbronchial biopsy or KVEIM test; and 3) abnormal lung function, either forced expiratory volume in one second (FEV1) or carbon monoxide diffusing capacity of the lung (DL,CO) $<75 \%$ of predicted normal value.

Patients were deemed ineligible for randomization if they had received a change in their dose of systemic steroids within a month of the start of the run-in period, or received any inhaled steroids within 6 weeks, and also if any of the following applied: current smoker, inability to complete the symptom record card or home lung function measurements, coexisting medical or psychiatric condition likely to affect or be adversely affected by participation in the trial, and anticipated need for heart-lung transplant within 1 yr.

Forty-four patients were recruited. One patient withdrew from the study before recording any data on the symptom record card or taking any of the study medication; intent to treat analysis was performed on the remaining 43 patients.

Patients who expressed an interest in the study were shown how to use a Diskhaler ${ }^{\mathrm{TM}}$ (Allen and Hanbuly's Ltd., Uxbridge, Middlesex, UK) and a Microspirometer ${ }^{\text {TM }}$ (Micromedical Ltd., Rochester, Kent, UK), and to complete symptom record cards. After an interval of 1-4 weeks, during which they used salbutamol as required by Diskhaler and became familiar with recording their symptoms and lung function measurements at home, the patients were randomized to either placebo (lactose only) or the study drug (FP $500 \mu \mathrm{g} \cdot \mathrm{blister}^{-1}$ ), two blisters twice daily via a Diskhaler for 6 months. Salbutamol $200 \mu \mathrm{g} \cdot$ blister $^{-1}$, was issued to all patients with a separate Diskhaler, for use as rescue medication as required. Treatment was continued for 6 months. Approximately $75 \%$ of the study population were receiving oral corticosteroids at the start of the study. The dose for each patient was optimized (and therefore variable between individuals), but then kept constant for at least six weeks before and during the 6-month study period. If rescue oral corticosteroids were required during the study, patients were excluded if they could no longer be controlled on their baseline medication.

All patients were asked to measure the following parameters (best of three readings on Microspirometer) once a week: peak expiratory flow rate (PEFR), FEV1, and forced vital capacity (FVC). In addition, they were asked to record on a four-point scale the severity of the following symptoms: breathlessness, cough, wheeze, general chest symptoms (all in the past week), sputum production (in the past $24 \mathrm{~h}$ ), and to note the number of salbutamol blisters used, other medication taken, and any other medical events, in the past week. Baseline symptom scores were the mean of the scores during the first week of the study. Trends of change within each treatment group were compared.

Clinic assessment was performed at $0,1,2,3$ and 6 months from randomization, and at a follow-up visit between 7 and 8 months. At all clinic visits, patients were examined and underwent spirometry (FEV1, FVC, and forced mid-expiratory flow (FEF25-75\%)) and gas transfer measurement ( $D \mathrm{~L}, \mathrm{CO}$ and carbon monoxide transfer coefficient $(K \mathrm{CO})$ ), and total lung capacity (TLC) in a lung function laboratory. At 0 and 6 months, patients completed the Health Status Questionnaire (Short Form (SF)-36), a measure of general health status [25].

Details of all adverse events (noted on symptom record cards or detected on clinical examination), and clinically significant changes in lung function or laboratory test results, were recorded on a structured report form, and the likelihood of these being due to the study medication was estimated by the investigator.

Blood was taken at each visit for measurement of haematological and biochemical variables, as well as serum glucose, unstimulated cortisol, and ACE levels. All samples were analysed centrally at the West Middlesex Laboratory, London, UK. An oropharyngeal swab was taken to exclude yeast infection at any visit if the patient complained of hoarseness or sore throat.

\section{Statistical analysis}

All statistical analyses were performed using SAS Version 6.08 (SAS Institute, Cary, NC, USA), with Proc GLM or FREQ used as appropriate. Comparisons between the two groups were made using analysis of covariance. All tests were two-sided, and a 5\% difference between groups was taken as statistically significant.

For clinic data, values obtained at the first visit were used as baseline. Patients (43 of the 44) with at least one diary card entry after randomization were included in the intent to treat analysis. Four patients (three on FP and one on placebo) did not return their symptom record cards, so these data were analysed for the remaining 39 patients.

For the record card symptom scores, each patient's median weekly score on a four-point scale was determined for each monthly time point, and the mean \pm SD of all patients' scores at this time point was calculated.

Correction of the results for lung disease classification, i.e. the proportions of patients with obstructive ( $\mathrm{FEV} 1 /$ FVC ratio $<80 \%$ pred) versus restrictive defects (FEV1/ FVC ratio $\geq 80 \%$ pred), did not substantially alter any of the results, which are therefore presented unadjusted.

\section{Results}

\section{Patient characteristics}

During a recruitment period of 18 months at the two centres, 44 patients were recruited, of whom one withdrew 
Table 1. - Patient characteristics

\begin{tabular}{lcc}
\hline & FP 2 mg· day $^{-1}$ & Placebo \\
\hline Patients n (male) & $21(7)$ & $22(10)$ \\
Ethnic origin n (\%) & $10(48)$ & $14(64)$ \\
Caucasian & $9(43)$ & $5(23)$ \\
Afro-Caribbean & $2(10)$ & $3(14)$ \\
Asian (not oriental) & $45 \pm 10$ & $48 \pm 11$ \\
Age yrs mean \pm SD & $28-65$ & $25-65$ \\
range & $4(18)$ & $1(5)$ \\
History of atopy n (\%) & $17(77)$ & $17(81)$ \\
Airways obstruction n (\%) & & \\
Duration of sarcoidosis yrs n & 4 & 1 \\
1-2 & 8 & 6 \\
3-5 $6-10$ & 4 & 8 \\
>10 & 5 & 15 \\
Concurrent medication n & 15 & \\
Oral corticosteroids & & 1 \\
B-gonist (short-acting & 0 & 18 \\
inhaled) & 0 & 13 \\
Methylxanthines & 15 & $12(7-16)$ \\
Other lung medication & 9 & \\
Nonlung medication & & \\
Baseline radiographic & $11(1-18)$ & \\
scores median (range) &
\end{tabular}

FP: fluticasone propionate. None of the differences were statistically significant.

from the study before recording any data, and the subsequent analyses were performed on the data from 43 patients on an intention to treat basis. Twenty-one patients were randomized to receive FP at $2 \mathrm{mg} \cdot$ day $^{-1} ; 22$ patients were randomized to the placebo group. Patient details are shown in table 1. At the time of the study, there were no significant differences between the study groups. All patients had abnormalities of lung function as defined in the study design. All patients had abnormal chest radiographs (grades II or III): in the FP group the median score was 11 (range 1-18) and in the placebo group 12 (7-16). The ethnic origin of the groups was varied and the majority had evidence of airways obstruction.

There were no significant differences in overall general health status, as measured by the SF-36, either across time or between the groups (data not shown). However, on one subscale of the SF-36, General Health Perception, the FP group showed a significant improvement between 0 and 6 months compared with the placebo group $(\mathrm{p}<0.02)$.

Mean \pm SD oral corticosteroid dosage did not change during the study for each group. In this regard, the oral corticosteroid dosage at entry for the placebo group was $5.71 \pm 5.41 \mathrm{mg} \cdot \mathrm{day}^{-1}$; at $1-3$ months $5.71 \pm 5.41$; and at 4-6 months 5.52 \pm 5.46 . The equivalent values for the FP group were: $6.74 \pm 6.46 ; 6.85 \pm 6.45$; and $5.92 \pm 6.66$, respectively.

Symptom score. In this group of patients who were selected because of apparent disease stability, thereby minimizing the confounding effects of changes in disease due to natural history, symptom scores of cough, breathlessness and wheeze were consistently lower in the group receiving FP at $2 \mathrm{mg}^{-\mathrm{day}^{-1}}$ at baseline during the 1-3 and 4-6 month time periods. The differences did not however achieve statistical significance (table 2).

Home recordings of peak expiratory flow rate and spirometry. The patients performed home spirometry and peak flow measurements on a weekly basis at the same time of day. No bronchodilators were to be used within $4 \mathrm{~h}$ of the measurements. The data from these home measurements are shown in table 2 and figure 1.

No changes were observed in either group in mean PEFRs expressed as summary measurements.

FEV1 was consistently higher in the FP group, and the differences between these groups was greatest during the two month follow-up period. These differences were not, however, statistically significant.

Clinic data. The comparison between clinical measurements of pulmonary function at baseline, 6 months and 2 months following the end of treatment for the two treatment groups are shown in table 3. From these it will be seen that PEFR changed very little during the treatment period, and there were no significant differences between the treatment groups. Similar data were found for measures of spirometry and gas transfer. In this regard, there were no differences in the change from baseline in FEV1, FVC, FEF25-75\%, TLC, DL,CO and $\mathrm{KCO}$. By chance the treatment group had higher

Table 2. - Record card data at 1-3 and 4-6 months for fluticasone propionate (FP) and placebo groups

\begin{tabular}{|c|c|c|c|c|c|c|}
\hline \multirow{2}{*}{ Parameter } & \multicolumn{3}{|c|}{ FP $2 \mathrm{mg} \cdot \mathrm{day}^{-1}$} & \multicolumn{3}{|c|}{ Placebo } \\
\hline & $\begin{array}{c}\text { Baseline } \\
(\mathrm{n}=18)\end{array}$ & $\begin{array}{c}1-3 \text { months } \\
(\mathrm{n}=18)\end{array}$ & $\begin{array}{l}\text { 4-6 months } \\
(\mathrm{n}=15)\end{array}$ & $\begin{array}{l}\text { Baseline } \\
(\mathrm{n}=21)\end{array}$ & $\begin{array}{c}1-3 \text { months } \\
(\mathrm{n}=21)\end{array}$ & $\begin{array}{c}\text { 4-6 months } \\
(\mathrm{n}=19)\end{array}$ \\
\hline \multicolumn{7}{|l|}{ Record card lung function } \\
\hline PEFR L $\cdot \min ^{-1}$ & $316 \pm 61$ & $319 \pm 65$ & $317 \pm 76$ & $319 \pm 130$ & $316 \pm 124$ & $322 \pm 124$ \\
\hline FEV1 L & $1.77 \pm 0.56$ & $1.85 \pm 0.58$ & $1.89 \pm 0.61$ & $1.62 \pm 0.57$ & $1.65 \pm 0.56$ & $1.76 \pm 0.63$ \\
\hline FVC L & $2.36 \pm 0.91$ & $2.36 \pm 0.86$ & $2.41 \pm 0.74$ & $2.14 \pm 0.74$ & $2.15 \pm 0.68$ & $2.31 \pm 0.74$ \\
\hline \multicolumn{7}{|l|}{ Record card symptom scores } \\
\hline Breathlessness & $0.89 \pm 0.76$ & $0.72 \pm 0.57$ & $0.73 \pm 0.59$ & $1.33 \pm 0.91$ & $1.14 \pm 0.85$ & $0.95 \pm 0.78$ \\
\hline Cough & $1.06 \pm 0.80$ & $0.89 \pm 0.83$ & $0.67 \pm 0.62$ & $1.14 \pm 0.85$ & $0.95 \pm 0.86$ & $0.68 \pm 0.89$ \\
\hline Wheeze & $0.42 \pm 0.58$ & $0.39 \pm 0.50$ & $0.47 \pm 0.64$ & $0.76 \pm 0.77$ & $0.52 \pm 0.75$ & $0.58 \pm 0.84$ \\
\hline Chest symptoms & $1.00 \pm 0.59$ & $0.89 \pm 0.58$ & $0.80 \pm 0.68$ & $1.14 \pm 0.91$ & $0.90 \pm 0.85$ & $0.74 \pm 0.81$ \\
\hline Sputum production & $0.68 \pm 0.68$ & $0.61 \pm 0.61$ & $0.47 \pm 0.64$ & $0.55 \pm 0.67$ & $0.43 \pm 0.60$ & $0.32 \pm 0.48$ \\
\hline Days of restricted activity $\cdot$ week $^{-1}$ & $0.03 \pm 0.12$ & $0.0 \pm 0.0$ & $0.0 \pm 0.0$ & $0.12 \pm 0.38$ & $0.0 \pm 0.0$ & $0.0 \pm 0.0$ \\
\hline Salbutamol blisters used-week ${ }^{-1}$ & $7.59 \pm 9.86$ & $8.8 \pm 11.7$ & $7.7 \pm 11.6$ & $4.87 \pm 13.3$ & $5.8 \pm 13.9$ & $7.1 \pm 15.2$ \\
\hline
\end{tabular}

Data are presented as means \pm SD. PEFR: peak expiratory flow rate; FEV1: forced expiratory volume in one second; FVC: forced vital capacity. Differences between FP and placebo were never significant. Neither group changed significantly in any parameter. 
a)

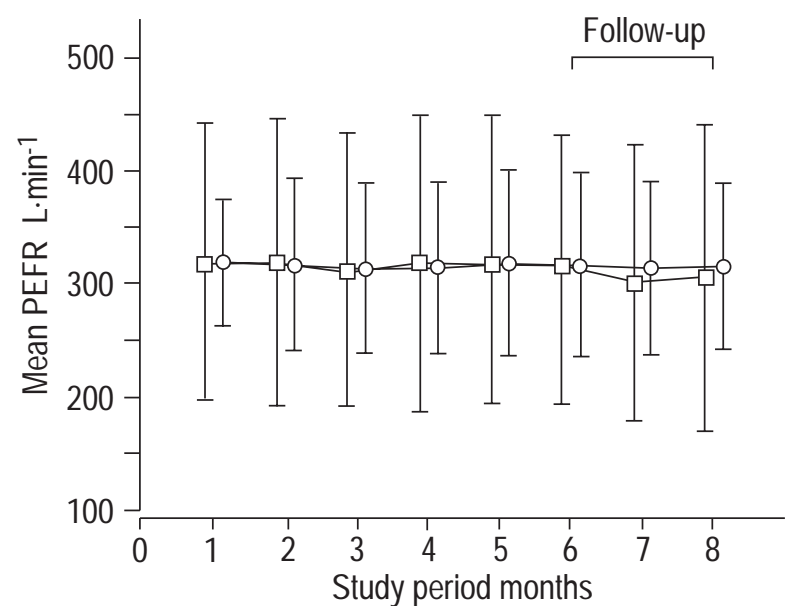

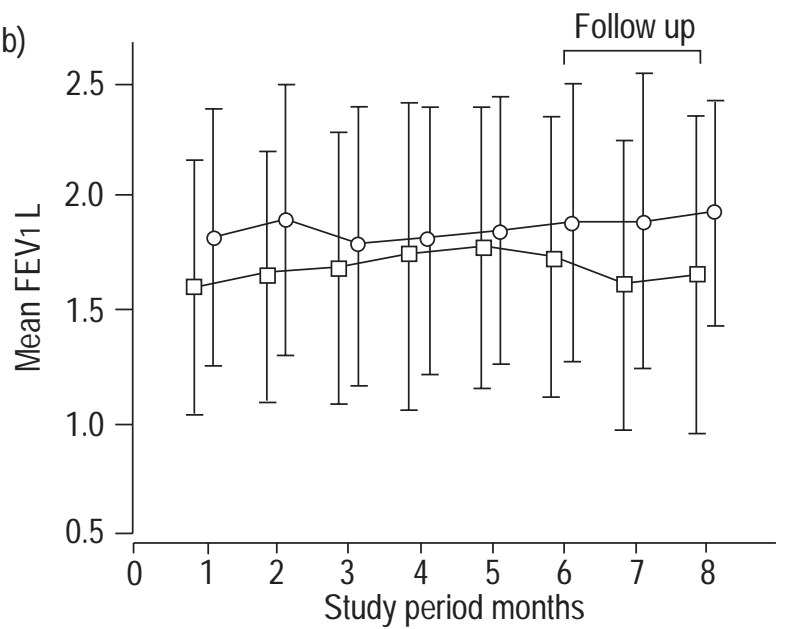

Fig. 1. - a) Peak expiratory flow rate (PEFR) and b) forced expiratory volume in one second (FEV1) summary measurements (mean \pm SD) in the fluticasone propionate $(\bigcirc ; 1 \mathrm{mg}$ b.i.d. via Diskhaler) and placebo $(\square)$ groups. Shown are mean data at each month during treatment and for 2 months of follow-up.

average values for all indices at presentation and thereafter, but the differential between the treated and placebo groups remained constant throughout the study.

Chest radiography. Radiological assessment at baseline and 6 months showed no significant differences between FP and placebo groups, and no differences across time in either group. On a score of 0 (no abnormality) to 3 (severe changes) assessed by two observers for three zones for each lung, there were no consistent changes in pattern or profusion between the two groups of patients. In the FP group median scores were 11 (range 1-18) pre-treatment and 11.5 (2-17) post-treatment. Corresponding scores for the placebo group were $12(7-16)$ and $11(6-17)$

\section{Safety}

Adverse events. There were no significant differences between the groups in the overall incidence of adverse events (reported by 18 out of 21 patients on FP and 19 out of 22 on placebo). The nature of the adverse events was similar in the two groups and was not unexpected for this patient population. The commonest events reported during treatment were upper respiratory infection (three patients on FP and seven on placebo), "asthma" (four patients on FP and six on placebo), chest infection (seven patients on FP and two on placebo), skin rash (two patients on FP and three on placebo), and hoarseness (four patients on FP). One patient on placebo (none on FP) developed oropharyngeal candidiasis at the third visit. In the posttreatment period, one patient on FP and three on placebo developed asthma, and one patient on FP developed a skin rash. There was no evidence of any clinically significant effect of FP on vital signs or laboratory parameters.

Changes in oral corticosteroid. Five patients (three on placebo, two on FP) required a temporary increase in oral corticosteroids but were included in the final intention to treat analysis.

Withdrawals from the study and serious adverse events. Six patients (four on FP and two on placebo) withdrew

Table 3. - Clinic data at baseline and after 6 months treatment for fluticasone propionate (FP) and placebo groups

\begin{tabular}{|c|c|c|c|c|c|c|}
\hline \multirow{2}{*}{ Parameter } & \multicolumn{3}{|c|}{ FP $2{\mathrm{mg} \cdot \text { day }^{-1}}^{-1}$} & \multicolumn{3}{|c|}{ Placebo } \\
\hline & $\begin{array}{c}\text { Baseline } \\
(\mathrm{n}=21)\end{array}$ & $\begin{array}{c}6 \text { months } \\
(\mathrm{n}=16)\end{array}$ & $\begin{array}{l}\text { Follow-up } \\
(\mathrm{n}=14)\end{array}$ & $\begin{array}{c}\text { Baseline } \\
(\mathrm{n}=22)\end{array}$ & $\begin{array}{c}6 \text { months } \\
(\mathrm{n}=18)\end{array}$ & $\begin{array}{l}\text { Follow-up } \\
(\mathrm{n}=14)\end{array}$ \\
\hline \multicolumn{7}{|l|}{ Clinic lung function data } \\
\hline PEFR $L \cdot \min ^{-1}$ & $355 \pm 74$ & $353 \pm 86$ & $357 \pm 89$ & $348 \pm 124$ & $338 \pm 125$ & $330 \pm 112$ \\
\hline FEV1 L & $2.00 \pm 0.65$ & $2.00 \pm 0.66$ & $2.00 \pm 0.67$ & $1.81 \pm 0.64$ & $1.80 \pm 0.63$ & $1.70 \pm 0.59$ \\
\hline FVC L & $2.98 \pm 1.09$ & $3.06 \pm 1.08$ & $2.91 \pm 1.07$ & $2.77 \pm 0.68$ & $2.87 \pm 0.67$ & $2.71 \pm 0.71$ \\
\hline FEF $25-75 \% \mathrm{~L}$ & $1.55 \pm 0.89$ & $1.31 \pm 0.76$ & $1.64 \pm 0.94$ & $1.23 \pm 0.92$ & $1.26 \pm 0.95$ & $1.24 \pm 1.03$ \\
\hline \multicolumn{7}{|l|}{ Clinic gas transfer data } \\
\hline$D \mathrm{~L}, \mathrm{CO}$ & $5.52 \pm 1.89$ & $5.57 \pm 2.26$ & $5.30 \pm 1.87$ & $5.36 \pm 1.46$ & $5.60 \pm 1.62$ & $5.31 \pm 1.51$ \\
\hline КСO & $1.55 \pm 0.41$ & $1.45 \pm 0.41$ & $1.53 \pm 0.45$ & $1.48 \pm 0.30$ & $1.48 \pm 0.33$ & $1.48 \pm 0.34$ \\
\hline TLC L & $4.33 \pm 1.44$ & $4.64 \pm 1.40$ & $4.28 \pm 1.34$ & $4.44 \pm 0.90$ & $4.72 \pm 0.78$ & $4.61 \pm 0.90$ \\
\hline $\mathrm{ACE}$ & $54.5 \pm 24.3$ & $52.5 \pm 41.2$ & $53.5 \pm 39.7$ & $83.3 \pm 38.9$ & $73.1 \pm 35.9$ & $90.3 \pm 53.9$ \\
\hline Serum cortisol level $\mathrm{nmol} \cdot \mathrm{L}^{-1}$ & $252 \pm 51$ & $226 \pm 64$ & $284 \pm 44$ & $271 \pm 45$ & $332 \pm 41$ & $317 \pm 45$ \\
\hline
\end{tabular}

Data presented as means \pm SD, except serum cortisol levels which are geometric means \pm SD. PEFR: peak expiratory flow rate; FEV1: forced expiratory volume in one second; FVC: forced vital capacity; FEF25-75\%: forced mid-expiratory flow; DL,CO: carbon monoxide diffusing capacity of the lung; KCO: carbon monoxide transfer coefficient; TLC: total lung capacity; ACE: angiotensin converting enzyme. Differences between FP and placebo were never significant. Neither group changed significantly from baseline. 
from the study because of adverse events, of which five were considered serious. The symptoms included: increasing breathlessness, wound breakdown, and increasing subcutaneous nodules in three patients on placebo. Only one of these was thought to be possibly drug-related before the study was unblinded. In the FP group, symptoms included dizziness and nausea associated with a fall in cortisol, and agitation, tachycardia and anxiety that were thought possibly to be drug-related. A third patient had abdominal and bone pain that were thought unlikely to have been caused by the drug.

Serum cortisol measurements. Values for serum cortisol levels are shown in table 3. No significant differences were found in overall change from baseline in each group. Seven patients who received FP and four who received placebo had low serum cortisol levels at some time during the study. The mean cortisol levels in the FP group were lower than the placebo group at 6 months. Two patients on FP had low cortisol levels at baseline; in four of the remainder (two in each group), levels returned to within threshold limits by the end of the study. There was no follow-up information on the remaining patients.

\section{Discussion}

This study was designed to assess the effects of FP in a dosage of $2 \mathrm{mg}^{- \text {day }^{-1}}$ on a number of clinical symptoms, physiological and radiological indices of sarcoidosis. A chronic disease group with stable sarcoidosis was deliberately chosen so that any variations in natural history which might confound the interpretation of the data were kept to a minimum. This pilot study showed no significant changes in lung function measurements made either at home or at serial clinic visits, nor was there any radiographic change. There were some differences in symptom scores and quality-of-life assessments, but only one of these was of a statistically significant value. Unfortunately the power of the study was almost certainly too low to detect a statistically significant difference in more comparisons, even though there was a tendency towards better symptomatic relief in the active treatment group.

The inhaled route for the administration of corticosteroids may be attractive as this limits systemic dose and the pathology is centred on lymphatic pathways, including peribronchially. This is shown in high resolution computed tomography (CT) imaging of the airways and also in histopathological studies where granulomas tended to be clustered around the bronchovascular bundles [26]. This attraction has been addressed by a number of studies over the years as to the effects of inhaled corticosteroids. Most of the previous studies did suggest some benefit, although most of these were not blinded or controlled. SELroos [11] studied patients which were defined as having active disease and found improvement in chest radiography and lung function indices. GUPTA [16] studied 113 patients who were divided into three groups, but no placebo or nontreatment groups were included. Objective indices did not change, but consistent with the present study and that of AlBERTS et al. [14], improvements in symptomatology were noted.
The trend towards improvement in symptom scores on patients' diary cards, and the significant improvement in the General Health Perception subscale of the SF-36 is consistent with the findings of ALBERTs et al. [14] in their randomized trial of BUD in newly-diagnosed pulmonary sarcoidosis, in which no differences were found in clinical, radiographic or most laboratory parameters, but the patients' Global Clinical Impression (GCI) score showed a significant difference in favour of the BUD group. Although no significant differences were found in symptom scores or measured indices, there were individuals who appear to benefit from FP. The numbers recruited however were too small for subgroup analysis.

It has been suggested that a subgroup of patients with both acute and chronic pulmonary sarcoidosis have marked bronchial hyperresponsiveness, and it may be this group who experience relief of symptoms, such as cough and dyspnoea, on treatment with BUD or FP [17]. Given the wide intra- and inter-individual variability in this condition, neither the present study nor that of AlBERTs et al. [14] was large enough to detect differences between the subgroups of patients with an obstructive versus restrictive pattern of disease. The patients in this study were a selected group in that, in addition to having had sarcoidosis for at least 12 months, none of them had taken inhaled steroids for at least six weeks prior to randomization. The patients would therefore have included some in whom inhaled steroids had been tried in the past and withdrawn because of lack of effect, and also those who were asked to discontinue inhaled steroids with a view to entering this trial. Patients who deteriorated in the six-week assessment period were not randomized but put back on their previous medication. Hence, the design of this trial was biased against certain subgroups of patients who might have responded to FP.

In conclusion, this study, which represents only the second blinded, placebo-controlled study of inhaled corticosteroids in sarcoidosis, adds to the understanding of disease modifying therapies by confirming that stable disease is not improved significantly physiologically or radiologically by treatment through this route. However, it is believed that the present selection process may have limited the opportunity for demonstrating benefit and that further longer period studies need to be undertaken of patients whose disease is less well established but persistent and therefore, possibly, more active. Such study designs can be constructed and need to be performed. The inhaled route will almost certainly be utilized for targeted treatment of lung conditions and more effective means of delivery should continue to be explored.

\section{References}

1. Scadding JG, Mitchell DN. Sarcoidosis. London, Chapman \& Hall, 1985.

2. Mangiapan G, Hance AJ. Mycobacteria and sarcoidosis: an overview and summary of recent molecular biological data (Review). Sarcoidosis 1995; 12; 20-37.

3. Spiteri MA, Newman SP, Clarke SW, Poulter LW. Inhaled corticosteroids can modulate the immunopathogenesis of pulmonary sarcoidosis. Eur Respir J 1989; 2: 218-224.

4. Siltzbach LE. Effects of cortisone in sarcoidosis: a study of thirteen patients. Am J Med 1952; 12: 139-160. 
5. Hunninghake GW, Gilbert S, Pueringer R, et al. Outcome of the treatment for sarcoidosis. Am J Respir Crit Care Med 1994; 149: 893-898.

6. Johns CJ, MacGregor MI, Zachery JB, Ball WC. Extended experience in the long-term corticosteroid treatment of pulmonary sarcoidosis. Ann NY Acad Sci 1976; 278: 722-731.

7. Israel HL, Fouts DW, Beggs RE. A controlled trial of prednisone treatment of sarcoidosis. Am Rev Respir Dis 1973; 107: 609-614.

8. Young RL, Harkerload LE, Lordon RE, Weg JG. Pulmonary sarcoidosis: a prospective evaluation of glucocorticoid therapy. Ann Intern Med 1970; 73: 207-212.

9. Turner-Warwick M, McAllister W, Lawrence R, Britten A, Haslam PL. Corticosteroid treatment in sarcoidosis: do serial lavage lymphocyte counts, serum angiotensin-converting enzyme measurements and gallium-67 scans help management? Thorax 1986; 41: 903-913.

10. Alberts C, Jansen HM, Roos CM, Out TA. Effects of inhaled budesonide in patients with pulmonary sarcoidosis. Eur Respir J 1991; 4: Suppl. 14, 253.

11. Selroos O. Budesonide in the treatment of pulmonary sarcoidosis. Sarcoidosis 1986; 3: 126-127.

12. Spiteri MA. Inhaled corticosteroids in pulmonary sarcoidosis. Postgrad Med J 1991; 67: 327-329.

13. Erikkilä S, Fröseth $\mathrm{B}$, Hellström $\mathrm{PE}$, et al. Inhaled budesonide influences cellular and biochemical abnormalities in pulmonary sarcoidosis. Sarcoidosis 1988; 5: 106110.

14. Alberts C, van der Mark ThW, Jansen HM and the Dutch Study Group on Pulmonary Sarcoidosis. Inhaled budesonide in pulmonary sarcoidosis: a double-blind, placebocontrolled study. Eur Respir J 1995; 5: 682-688.

15. Milman N, Graudal N, Grode G, Munch E. No effect of high-dose inhaled steroids in pulmonary sarcoidosis: a double-blind, placebo-controlled study. $J$ Intern Med 1994; 236: 285-290.

16. Gupta SK. Treatment of sarcoidosis patients by steroid aerosol: a ten year prospective study from Eastern India. Sarcoidosis 1989; 6: 51-54.

17. Kirsten D. Inhaled steroids for sarcoidosis? Eur Respir $J$ 1995; 8: 679-681.

18. Shaw RJ. Pharmacology of fluticasone propionate. Respir Med 1994; 88: Suppl A, 5-8.

19. Meltzer EO, Orgel HA, Rogenes PR, Field EA. Nasal cytology in patients with allergic rhinitis: effects of intranasal fluticasone propionate. $J$ Allergy Clin Immunol 1994; 94: 708-715.

20. Chervinsky P, van As A, Bronsky EA, et al. Fluticasone propionate aerosol for the treatment of adults with mild to moderate asthma. The Fluticasone Propionate Asthma Study Group. J Allergy Clin Immunol 1994; 94: 676-683.

21. Dahl R, Lundback B, Malo JL. A dose-ranging study of fluticasone propionate in adults with moderate asthma. International Study Group. Chest 1993; 104: 1352-1358.

22. Greening AP. Clinical trials of high-dose fluticasone dipropionate. Respir Med 1994; 88: Suppl A, 17-23.

23. Holliday SM, Faulds D, Sorkin EM. Inhaled fluticasone propionate. A review of its pharmacokinetic properties, and therapeutic use in asthma. Drugs 1994; 47: 318-331.

24. Llewellyn-Jones CG, Hill SL, Stockley RA. Effect of fluticasone propionate on neutrophil chemotaxis, superoxide generation, and extracellular proteolytic activity in vitro. Thorax 1994; 49; 207-212.

25. Anderson RT, Aaronson NK, Wilkin D. Critical review of the international assessments of health-related quality of life. Qual Life Res 1993; 2: 369-395.

26. Muller NL, Kullnig P, Miller RR. The CT findings of pulmonary sarcoidosis: analysis of 25 patients. Am J Roent 1989; 152: 1179-1182. 